\title{
COVID-19 Guideline Modifications as CMS Announces "Opening Up America Again": Comments from the Society of Surgical Oncology
}

\author{
James R. Howe, MD', David L. Bartlett, $\mathrm{MD}^{2}$, Douglas S. Tyler, MD ${ }^{3}$, Sandra L. Wong, $\mathrm{MD}^{4}$, \\ Kelly K. Hunt, $\mathrm{MD}^{5}$, and Ronald P. DeMatteo, $\mathrm{MD}^{6}$ for the Society of Surgical Oncology \\ ${ }^{1}$ University of Iowa Hospitals and Clinics, Iowa City, IA; ${ }^{2}$ University of Pittsburgh Medical Center, Pittsburgh, PA; \\ ${ }^{3}$ University of Texas Medical Branch, Galveston, TX; ${ }^{4}$ Dartmouth-Hitchcock Medical Center, Lebanon, NH; ${ }^{5}$ University \\ of Texas MD Anderson Cancer Center, Houston, TX; ${ }^{6}$ Hospital of the University of Pennsylvania, Philadelphia, PA
}

On 19 April 2020, the Center for Medicare and Medicaid Services (CMS) released a document entitled "Opening Up America Again: Centers for Medicare \& Medicaid Services (CMS) Recommendations for Reopening Facilities to Provide Non-emergent Non-COVID19 Healthcare: Phase I." ${ }^{1}$ The CMS recognizes that some areas of the United States have low and/or stable numbers of COVID-19 patients, and that healthcare facilities should have greater flexibility to deliver care currently deferred due to the pandemic, including surgical procedures. Regions must fulfill what the White House has defined as "gating criteria" to allow progress to phase 1 of re-opening. ${ }^{2}$ These criteria include:

1. A downward trajectory of cases with influenza-like illness or COVID-like symptoms within 14 days

2. A downward trajectory of documented COVID-19 cases or positive tests (as a percentage of total tests) within 14 days

3. Hospitals are treating patients without crisis care

4. Robust testing programs are in place for at-risk health care workers.

The authors are members of the 2020-2021 Executive Committee of the Society of Surgical Oncology.

(C) Society of Surgical Oncology 2020

First Received: 27 April 2020;

Published Online: 6 May 2020

J. R. Howe, MD

e-mail: james-howe@uiowa.edu
Phase 1 of re-opening would allow resumption of elective surgeries as clinically appropriate on an outpatient basis at facilities that adhere to CMS guidelines. If a region shows no rebound in the number of cases and satisfies the 14-day gating criteria a second time, it can move to phase 2 , in which "elective surgery can resume, as clinically appropriate, on an out- or inpatient basis at facilities that adhere to CMS guidelines." 2 If a region then shows no rebound in the number of cases and satisfies the 14-day gating criteria a third time, it can move to phase 3 , in which surgery can fully resume, and other social restrictions can be relaxed (e.g., unrestricted staffing of worksites, limited physical distancing in large venues).

The CMS document suggests that "providers should prioritize surgical/procedural care and high/complexity chronic disease management." capacity, a healthy workforce, adequate personal protective equipment (PPE), and post-acute care that would not jeopardize the facility's capacity to respond to another surge in COVID-19 cases. Facilities also should continue taking steps to reduce transmission (distancing, separation of COVID-19-free spaces, prohibition of visitors, increased sanitation protocols), and all patients should be screened for symptoms and by laboratory testing "before care" (presumably including surgery). Health care workers also should be regularly screened by laboratory testing when "adequate testing capability is established."

To summarize, the White House and CMS documents ${ }^{1,2}$ suggest that facilities with down-trending numbers of COVID-19 cases, adequate testing abilities, and no shortages of PPE, intensive care unit (ICU) beds, ventilators, or health care workers may be able to resume elective surgeries, which would reasonably include all cancer cases. 
Phase 1 of recovery, as described by the White House document, would allow outpatient procedures for cancer patients, which had been deferred as lower-priority operations during the pandemic phase of care. Phase 2 then would allow for cases requiring inpatient care and for outpatient procedures. In their document, "Local Resumption of Elective Surgery Guidance," the American College of Surgeons (ACS) also has given detailed suggestions about what facilities should do to prepare for the ramping up needed for initiation of elective surgeries. ${ }^{3}$

The ACS recently updated their cancer-triaging suggestions during COVID-19 to include a recovery phase in a document entitled "ACS Guidelines for Triage and Management of Elective Cancer Surgery Cases During the Acute and Recovery Phases of Coronavirus Disease 2019 (COVID-19) Pandemic." "This document breaks down the COVID-19 outbreak into the pandemic phases for which the Society of Surgical Oncology (SSO $)^{5}$ and the $\mathrm{ACS}^{6}$ had already posted guidelines (on 24 March 2020) for triaging of cancer cases, and these new guidelines now include two recovery phases. The early recovery phase is characterized by fewer COVID-19 cases each day and greater availability of limited resources such as PPE, health care workers, ventilators, ICU beds, and testing. In the late recovery phase, the facility is more than 14 days beyond its peak, and resources are at near normal levels. The ACS document ${ }^{4}$ gives specific suggestions for prioritizing cancer cases in the acute and late recovery phases for patients with breast cancer, colorectal cancer, thoracic malignancies, pancreatic and periampullary cancers, soft tissue sarcoma, and melanoma.

Although the release of these documents from the White House and the $\mathrm{CMS}^{1,2}$ are encouraging for surgeons, inspiring hope that they may be able to resume elective surgeries soon, all regions of the country and even specific hospitals within the same region will have unique challenges in meeting these proposed criteria. Some confusion may result from the fact that phase 1 of recovery mentions only performance of outpatient procedures, and that individual states may have different criteria mandated by their governors. Therefore, surgeons must work closely with their hospital leadership and local authorities to determine whether they fall within the pandemic or recovery phases, and whether they meet gating criteria as well as CMS and state guidelines. If these standards are met, then it would be reasonable for hospitals to resume elective surgeries for cancer patients, which could include both in- or outpatient procedures because few cancer cases would be considered as truly elective.

The updated ACS guidelines for triage give detailed suggestions on how to prioritize cancer cases that have been deferred at these six disease sites. ${ }^{4}$ A general principle emerging from these suggestions is that clinicians must review the priority of cancer cases suggested in the pandemic phases, and begin by performing the more urgent cases that were delayed, followed by the semi-urgent cases. Afterward, other cancer cases can follow based on prioritization as to which patients are most likely to have compromised outcomes with further delays. It is important for health care workers to be aware that there could be a resurgence of COVID-19 cases related to seasonal changes (in the fall or winter), as social distancing practices are relaxed, or as a result of other currently unforeseen factors. Should this happen, these events could again lead to severe restrictions in cancer care delivery and a return to these triage guidelines for cancer patients. All guidelines will also need to be updated periodically as both COVID-19 polymerase chain reaction and antibody testing become more universally available, effective drugs are identified, and/or a successful vaccine is developed.

Readers might also be interested in how the COVID-19 pandemic is affecting the academic mission in surgical oncology, ${ }^{8}$ and a detailed example of how one medical center has navigated the issues surrounding COVID-19.9

DISCLOSURES Dr. Kelly K. Hunt reports medical advisory board support from Armada Health and Merck \& Co.; research funding to her institution from Endomagnetics, Lumicell, and OncoNano. All other authors report no conflicts.

\section{REFERENCES}

1. Center for Medicare \& Medicaid Services. Opening Up America Again: Centers for Medicare \& Medicaid Services (CMS) Recommendations for Re-opening Facilities to Provide Nonemergent Non-COVID-19 Healthcare: Phase I. Retrieved 24 April 2020 at https://www.cms.gov/files/document/covid-flexibility-reo pen-essential-non-covid-services.pdf.

2. The Whitehouse, Centers for Disease Control and Prevention. Guidelines: Opening Up America Again. Retrieved 24 April 2020 at https://www.whitehouse.gov/openingamerica/-criteria.

3. American College of Surgeons. Local Resumption of Elective Surgery Guidance. Retrieved 24 April 2020 at https://www.facs. org/covid-19/clinical-guidance/resuming-elective-surgery.

4. American College of Surgeons. ACS Guidelines for Triage and Management of Elective Cancer Surgery Cases During the Acute and Recovery Phases of Coronavirus Disease 2019 (COVID-19) Pandemic. Updated 24 April 2020. Retrieved 24 April 2020 at h ttps://www.facs.org/-/media/files/covid19/acs_triage_and_manage ment_elective_cancer_surgery_during_acute_and_recovery_phase s.ashx.

5. Bartlett DL, Howe JR, Chang G, et al. Management of cancer surgery cases during the COVID-19 pandemic: considerations. Ann Surg Oncol. 2020. https://doi.org/10.1245/s10434-020-084612.

6. American College of Surgeons. COVID-19 Guidelines for Triage of Cancer Surgery Patients. Retrieved 24 April 2020 at https:// www.facs.org/covid-19/clinical-guidance/elective-case/cancer-sur gery.

7. The COVID19 Subcommittee of the O.R. Executive Committee at Memorial Sloan Kettering. Cancer surgery and COVID19. Ann Surg Oncol. 2020. https://link.springer.com/article/10.1245/s1043 4-020-08462-1. 
8. Pawlik TM, Tyler DS, Sumer B, et al. COVID-19 pandemic and surgical oncology: preserving the academic mission. Ann Surg Oncol. 2020. https://doi.org/10.1245/s10434-020-08563-x.

9. Walker JP. Resuming Elective Surgery at UTMB Predicated on Patient and Staff Well-being. Retrieved 24 April 2020 at https:// www.facs.org/-/media/files/covid19/resuming_elective_surgery_at _utmb.ashx.
Publisher's Note Springer Nature remains neutral with regard to jurisdictional claims in published maps and institutional affiliations. 